The Astrophysical JouRnAL, 497:330-341, 1998 April 10

(C) 1998. The American Astronomical Society. All rights reserved. Printed in U.S.A.

\title{
CANDIDATE MAIN-SEQUENCE STARS WITH DEBRIS DISKS: A NEW SAMPLE OF VEGA-LIKE SOURCES
}

\author{
VINCENT MANNINGS \\ Division of Physics, Mathematics and Astronomy, California Institute of Technology, MS 105-24, Pasadena, CA 91125 \\ AND
}

Michael J. BARLOW

Department of Physics and Astronomy, University College London, Gower Street, London WC1E 6BT, UK Received 1996 July 24; accepted 1997 November 17

\begin{abstract}
Vega-like sources are main-sequence stars that exhibit IR fluxes in excess of expectations for stellar photospheres, most likely due to reradiation of stellar emission intercepted by orbiting dust grains. We have identified a large sample of main-sequence stars with possible excess IR radiation by crosscorrelating the Michigan Catalog of Two-dimensional Spectral Types for the HD Stars with the IRAS Faint Source Survey Catalog. Some 60 of these Vega-like sources were not found during previous surveys of the IRAS database, the majority of which employed the lower sensitivity Point Source Catalog. Here, we provide details of our search strategy, together with a preliminary examination of the full sample of Vega-like sources.
\end{abstract}

Subject headings: circumstellar matter - infrared: stars

\section{INTRODUCTION}

Vega-like sources are main-sequence stars with disks or rings of orbiting dust grains. These circumstellar structures may be the evolved remnants of once massive disks, perhaps augmented by debris products generated during the ongoing disruption of planetesimal-sized bodies (cf., Backman \& Paresce 1993, and references therein). The disks represent an important end state for material that has survived - and is being replenished - long after the termination of the protostellar collapse phase, and beyond the (presumed) era of planet formation, too. The detailed study of the composition, size, and spatial distribution of the dust grains comprising these disks therefore promises rich insight to the processes by which planetary systems are created.

Aumann (1985) restricted the adjective "Vega-like" to main-sequence stars with flux densities at $60 \mu \mathrm{m}$ that are in excess of the levels expected for stars of their spectral types, as evidenced during all-sky survey measurements with the Infrared Astronomical Satellite (IRAS). We will use the term "Vega-like" more flexibly here to describe a main-sequence star that exhibits excess flux at any IRAS infrared (IR) wavelength.

The eponymous star Vega ( $\alpha$ Lyr) was serendipitously found by Aumann et al. (1984) to be approximately 1 order of magnitude brighter at far-IR wavelengths than expected for an A0 V star. The additional flux was ascribed to radiation from grains in thermal equilibrium with the stellar radiation field, and distributed within a shell or ring with an inner radius of several tens of AU. Shortly after this discovery the A5 V star $\beta$ Pic, also found to have excess far-IR emission (Gillett 1986), was observed with an optical coronagraph by Smith \& Terrile (1984), revealing a highly inclined dust-scattering disk extending out to hundreds of AU from the star.

The IRAS measurements were the first to identify excess IR emission from main-sequence stars that are not undergoing significant mass loss from their surfaces. Subsequently, many programs of study have sought to understand the Vega-like stars within the context of the end products of the evolution of circumstellar disks associated with pre-main-sequence stars, the possible formation of planetesimals and planets, and perhaps even the disruption of infalling cometary bodies. See the reviews by Backman \& Paresce (1993) and Lagrange-Henri (1995), and see also Vidal-Madjar \& Ferlet (1994). Despite attempts to image the material around many other Vega-like sources (e.g., Smith, Fountain, \& Terrile 1992; Kalas \& Jewitt 1996), only one further star, BD $+31^{\circ} 643$ (Kalas \& Jewitt 1997) has joined $\beta$ Pic in the select group for which the circumstellar material has been imaged directly at optical wavelengths. (Skinner et al. 1995 have imaged a dust structure around SAO 26804 at $\lambda \approx 10 \mu \mathrm{m}$.) Our information on the nature of most Vega-like sources has instead been gleaned indirectly from ground-based optical and near- and mid-IR spectroscopy and by combining the IRAS measurements with ground-based near-IR and millimeter-wave photometry for studies of the continuum spectral energy distributions. In these ways the composition of grains, together with their temperature, size, spatial distribution, and total mass, have all been probed (Chini et al. 1991; Telesco \& Knacke 1991; Skinner, Barlow, \& Justtanont 1992; Aitken et al. 1993; Knacke et al. 1993; Zuckerman \& Becklin 1993; Sylvester, Barlow, \& Skinner 1994a, 1994b; Skinner et al. 1995; Sylvester et al. 1996).

At the same time, various searches of the IRAS catalogs have been made to try to identify new candidate Vega-like stars (see below for a summary). In the present work we provide the results of a new search of the IRAS Faint Source Survey Catalog (Moshir et al. 1989) for associations of IR sources with Southern Hemisphere main-sequence stars listed in the current version of the Michigan Catalog of Two-dimensional Spectral Types for the HD Stars. Multiband near- to far-IR photometry of a subset of these Vega-like candidates is being obtained using the Infrared Space Observatory (ISO), and we have an ongoing program of ground-based observations. These measurements will be discussed in a forthcoming set of papers. Here we provide 
preliminary details of the full set of Vega-like stars identified in the Michigan catalog. In $\S 2$ we briefly describe earlier searches of the IRAS data. The database employed for the present work is described in $\S 3$, together with the criteria used to select Vega-like sources and the method employed to identify excess mid- and far-IR fluxes. The results are tabulated and described in $\S 4$. Our search method is compared in $\S 5$ to those employed in some previous surveys of the $I R A S$ catalogs, and we discuss our results in $\S 6$.

\section{PREVIOUS SURVEYS}

At least a dozen searches have been made through the IRAS database for evidence of IR sources associated with samples of objects that include stars on the main sequence. These are summarized in Table 1. Most employed the IRAS Point Source Catalog, which provides flux densities measured in four wavelength bands centered approximately at $12,25,60$, and $100 \mu \mathrm{m}$, and were limited to particular spectral types and to bright and/or nearby stars. We will briefly mention the larger surveys here.

The first major search (Walker \& Wolstencroft 1988) used both the full SAO catalog and the Catalog of Nearby Stars (Gliese 1969), and included the selection criterion that potential candidates have a 60:100 $\mu \mathrm{m}$ flux density ratio similar to that of the prototype Vega-like stars. All selected sources were further required to exhibit evidence for extended emission in one or more of the IRAS photometric bands. Of the resulting set of just 34 stars, only half are known to be on the main sequence. By relaxing both these restrictions, Stencel \& Backman (1991) identified a total of 379 SAO stars with apparent IR excesses but, again, mainsequence dwarfs comprised only a subset. (See also § 5 below). Oudmaijer et al. (1992) made a similar survey of all luminosity classes, but with the exclusion of stars with spectral type K and later. (See § 5). Backman \& Paresce (1993) have compiled a list of 54 main-sequence stars with midand far-IR excesses, culled from several of the surveys noted in Table 1.

\section{THE NEW SEARCH FOR VEGA-LIKE SOURCES}

\subsection{The Database}

The aim of the present work is to make a systematic search for main-sequence stars with IR excesses using the
IRAS Faint Source Survey Catalog (Moshir et al. 1989, hereafter FSC), which contains information on 173,044 sources in the relatively unconfused regions of sky at Galactic latitudes $|b| \geq 10^{\circ}$, corresponding to approximately $80 \%$ of the sky. The FSC has the best limiting sensitivity of all the available IRAS data products and is approximately 1 mag more sensitive than the IRAS Point Source Catalog, Version 2, 1988 (hereafter PSC) as a result of co-adding the full set of point-source-filtered individual detector scans of any given sky region, in each of the four wavelength bands.

To work with a large sample of stars with accurately determined luminosity classifications, we have used digitized versions of the four released volumes of the Michigan Catalog of Two-dimensional Spectral Types for the HD Stars, which we shall henceforth refer to as the Michigan Spectral Catalog (MSC: Houk \& Cowley 1975; Houk 1978, 1982; Houk \& Smith-Moore 1988; Houk 1994). This ongoing survey provides information on the spectral types and luminosity classes - the two "dimensions" in the name of the catalog - of some 130,397 HD stars in the declination range $\delta=-90^{\circ}$ to $-12^{\circ}$. Although the four volumes of the MSC published to date cover less than $50 \%$ of the sky, they include $58 \%$ of the stars in the HD catalog (Houk 1994), due to the offset of the Galactic center to southern declinations. This potential advantage is, however, offset by the likelihood that the majority of the main-sequence stars found by our sampling method are relatively nearby, so that Volumes 1-4 of the MSC may only contain about $50 \%$ (or less) of the main-sequence stars having infrared excesses that are potentially extractable from the IRAS FSC. For each star, the MSC records the designation in the HD catalog, the newly determined spectral type and luminosity class, the photographic $V$-band magnitude, and the equatorial and Galactic coordinates. Equatorial coordinates are provided for equinox 1900. Also given are the centennial precessions in right ascension and declination, which we have used to transform all equatorial coordinates to equinox 1950, for consistency with the equinox adopted for the IRAS catalogs. (Spot checks of the results of these transformations were made by comparing to corresponding entries in the SAO catalog - equinox 1950.0 - and the precessed coordinates were found to be consistent.) The equatorial coordinates listed in Volume 1 of the MSC (Houk \& Cowley 1975) are taken directly from the original HD catalog and are not sufficiently precise for our purposes:

TABLE 1

Previous Surveys of the iRAS Data Catalogs

\begin{tabular}{|c|c|c|}
\hline Authors & Database & Comments \\
\hline Aumann $1985 \ldots \ldots \ldots \ldots \ldots \ldots \ldots$ & PSC, ${ }^{\mathrm{a}}$ Catalog of Nearby Stars & Identified eight new Vega-like stars \\
\hline Jaschek, Jaschek, \& Egret $1986 \ldots \ldots$. & PSC, Catalog of Stellar Groups & Ae/A-type shell stars only \\
\hline Johnson $1986 \ldots \ldots \ldots \ldots \ldots \ldots \ldots \ldots$ & PSC, compilation of dKe stars & K Ve stars only \\
\hline Odenwald $1986 \ldots \ldots \ldots \ldots \ldots \ldots \ldots \ldots$ & PSC, various compilations & G-type stars only, including dwarfs \\
\hline Sadakane \& Nishida 1986 ........... & PSC, Bright Star Catalog & 12 new Vega-like stars identified \\
\hline Coté $1987 \ldots \ldots \ldots \ldots \ldots \ldots \ldots \ldots \ldots \ldots$ & PSC, Bright Star Catalog & B and A stars only \\
\hline Walker \& Wolstencroft 1988 ........ & PSC, SAO, Catalog of Nearby Stars & Search for sources with IRAS colors of prototypes \\
\hline Aumann \& Probst $1991 \ldots \ldots \ldots \ldots \ldots$ & PSC, Catalog of Nearby Stars & Search for $12 \mu \mathrm{m}$ excesses \\
\hline Patten \& Willson 1991 .............. & $\begin{array}{l}\text { PSC, MSC, Bright Star Catalog, Catalog of } \\
\text { Stellar Rotational Velocities }\end{array}$ & Main-sequence $\mathrm{B}, \mathrm{A}$, and $\mathrm{F}$ stars \\
\hline Stencel \& Backman $1991 \ldots \ldots \ldots \ldots .$. & PSC, SAO & $\begin{array}{l}\text { Included } \mathrm{B}, \mathrm{A}, \mathrm{F}, \mathrm{G}, \mathrm{K} \text {, and } \mathrm{M} \text { stars of all } \\
\text { luminosity classes }\end{array}$ \\
\hline Cheng et al. $1992 \ldots \ldots \ldots \ldots \ldots \ldots \ldots$ & $\begin{array}{l}\text { FSC, Bright Star Catalog, Catalog of Stars } \\
\text { within } 25 \mathrm{pc} \text { of the Sun }\end{array}$ & Main-sequence A-type stars only \\
\hline Oudmaijer et al. $1992 \ldots . .$. & PSC, SAO & Included $\mathrm{B}, \mathrm{A}, \mathrm{F}$, and $\mathrm{G}$ stars of all luminosity classes \\
\hline
\end{tabular}

${ }^{\text {a }}$ IRAS Point Source Catalog. 
right ascension is given to an accuracy of 0.1 minute of time, and declination to $1^{\prime}$. (Volumes 2-4 provide positions that are accurate to $0.1 \mathrm{~s}$ in right ascension, and to $1^{\prime \prime}$ in declination.) We have cross-correlated the records in Volume 1 (according to HD designation) with the SAO catalog in order to exploit the accurate coordinates provided by the latter. The resulting subset $(52 \%)^{1}$ of Volume 1 stars is used together with Volumes 2-4 throughout the present work, providing a combined set of 112,971 stars.

\subsection{Procedure for Identification of Sources}

To be considered as Vega-like candidates, associations of MSC stars with FSC IR sources are required to satisfy the following criteria:

1. The separation on the sky must be $\leq 60^{\prime \prime}$. As a result, $83 \%$ of MSC stars are rejected, leaving 19,480 in total.

2. The quality of the IRAS flux densities of the positionassociated sources must be flagged in the FSC as either "excellent" or "moderate," which correspond respectively to "FQUAL" flags 3 and 2. (FQUAL $=1$ signifies a nondetection.) To avoid spurious associations with Galactic "cirrus" emission at 60 and $100 \mu \mathrm{m}$, we accept only candidates with excellent/moderate detections in one of the following combinations of wavelength bands: (a) 12,25 , and $60 \mu \mathrm{m}(b) 12$ and $25 \mu \mathrm{m}(c) 25$ and $60 \mu \mathrm{m}$. On these criteria, a further 14,158 MSC stars are rejected, producing a subset of 5322 sources.

3. To restrict ourselves to main-sequence stars, we next reject those sources with MSC luminosity classifications in the range I-IV. A small subset of $294 \mathrm{MSC} / \mathrm{FSC}$ candidates for which the MSC claims a luminosity class of $\mathrm{V}$ then remains. Note that we exclude from this subset all sources for which a main-sequence classification is uncertain, i.e., those classed in the MSC as III/V or IV/V.

4. Finally, we are left with just 131 stars after rejecting 163 luminosity class V MSC stars that fail to exhibit significant IR flux excesses above the photospheric values expected at or longward of $\lambda=12 \mu \mathrm{m}$. The scheme for defining these excesses is described below.

\subsection{Method for Testing for Excess IR Emission}

Let the 12, 25, and $60 \mu \mathrm{m}$ flux densities listed in the FSC for any given source be $F_{12}, F_{25}$, and $F_{60}$, respectively. We consider the two ratios of flux densities defined by

$$
R_{12 / 25} \equiv \frac{F_{12}}{F_{25}}
$$

and

$$
R_{25 / 60} \equiv \frac{F_{25}}{F_{60}} .
$$

By comparing the ratios of measured flux densities to the ratios expected for a star of given spectral type, we have a simple and straightforward method for automating the rejection of those stars without significant mid- to far-IR

\footnotetext{
${ }^{1} 18,956$ stars from the 36,382 stars of Volume 1.
}

excesses. ${ }^{2}$ We know from the FSC the (independent) uncertainties, $\delta F$, on each of the IRAS flux densities, so that we immediately obtain an expression for the error on the observed 12:25 $\mu \mathrm{m}$ ratio:

$$
\delta R_{12 / 25}= \pm \frac{F_{12}}{F_{25}} \sqrt{\left(\frac{\delta F_{12}}{F_{12}}\right)^{2}+\left(\frac{\delta F_{25}}{F_{25}}\right)^{2}} .
$$

Similarly,

$$
\delta R_{25 / 60}= \pm \frac{F_{25}}{F_{60}} \sqrt{\left(\frac{\delta F_{25}}{F_{25}}\right)^{2}+\left(\frac{\delta F_{60}}{F_{60}}\right)^{2}} .
$$

We compute expected photospheric ratios $\left(R_{12 / 25}^{*}\right.$ and $R_{25 / 60}^{*}$ ) using the MSC spectral types and blackbodies corresponding to the stellar effective temperatures determined by Gray \& Corbally (1994). Discrepancies at significance levels greater than $1 \sigma$ between the observed and the expected ratios therefore occur when

$$
\frac{\Delta_{12 / 25}}{\delta_{\text {obs }}} \equiv \frac{R_{12 / 25}-R_{12 / 25}^{*}}{\delta R_{12 / 25}}<-1
$$

and/or

$$
\frac{\Delta_{25 / 60}}{\delta_{\text {obs }}} \equiv \frac{R_{25 / 60}-R_{25 / 60}^{*}}{\delta R_{25 / 60}}<-1 .
$$

In other words, we claim that one or more of the observed flux densities significantly exceed expectation for a dust-free stellar photosphere if either or both of the inequalities in equations (5) and (6) are satisfied. (We do not use any flux density with an FSC quality flag of 1, regardless of the ratio of the flux density and its listed $1 \sigma$ uncertainty.) Note that we implicitly assume that all MSC spectral types are free of error, so that

$$
\delta R_{12 / 25}^{*}=\delta R_{25 / 60}^{*}=0 .
$$

We note that the blackbody photospheric flux ratios that we adopted for our comparisons correspond to monochromatic flux density ratios at the standard wavelengths of the IRAS filters, rather than to the in-band flux ratios for the IRAS filters. However, for a $5000 \mathrm{~K}$ blackbody the 12 and $25 \mu \mathrm{m}$ monochromatic flux ratio is 4.084 , while the in-band flux ratio is 4.172 (IRAS Explanatory Supplement 1988), which differ by only by a factor of 1.021 , while for a 10,000 $\mathrm{K}$ blackbody the ratios are 4.225 and 4.345 , differing by only a factor of 1.028 . Not only are these differences of less than $3 \%$ between the blackbody monochromatic and in-band flux ratios very much smaller than the deviations exhibited by the sources found to have excesses (see Fig. 1), but our requirement for an excess relative to blackbody monochromatic flux density ratios is more conservative than comparing to blackbody in-band flux ratios, since slightly more emission is required from a star at the longer wavelength before it is classified as having an excess. The same remarks apply to the $25 / 60 \mu$ m blackbody flux ratio criterion. We also note that Kurucz LTE model atmospheres for A-type stars predict photospheric infrared energy distributions that are even slightly more steep than for blackbodies, e.g., the $9850 \mathrm{~K}, \log g=4.25$ model for Sirius presented by Cohen et al. (1992) predicts $12 / 25 \mu \mathrm{m}$

\footnotetext{
${ }^{2}$ Only raw FSC flux densities are used: i.e., we attempt no bandpass color corrections in the absence of prior knowledge of the temperature distribution of all material contributing to any excess infrared emission from a given source.
} 


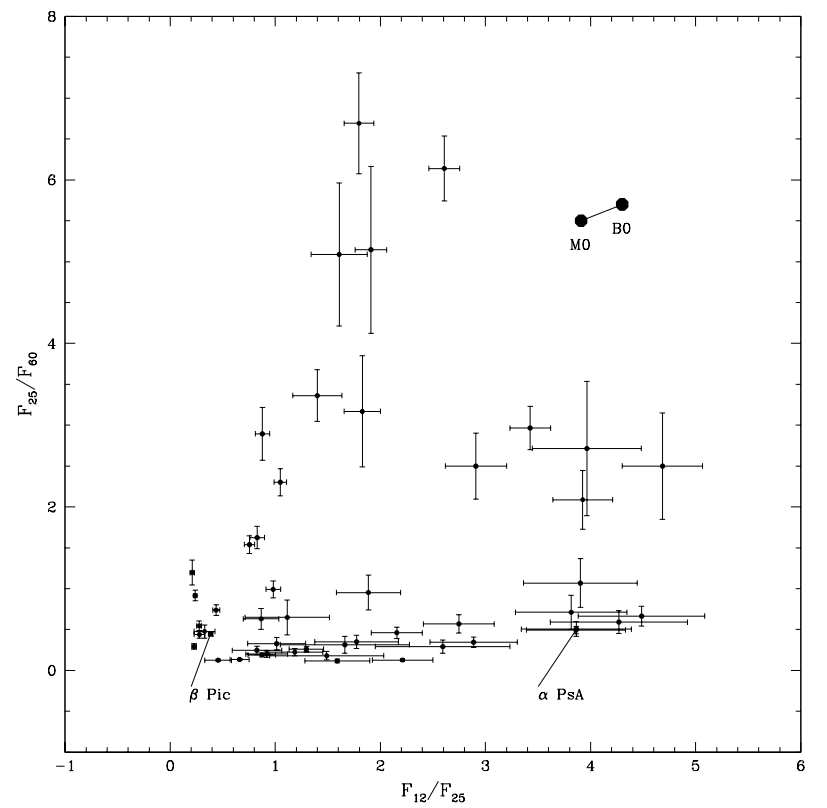

FIG. 1.-Diagrammatic comparison of flux density ratios for all sources (new and previously recognized) that have excellent- or moderate-quality FSC measurements in each of the three 12, 25, and $60 \mu \mathrm{m}$ bands, and for which at least one of the ratios departs from the photospheric value at the $3 \sigma$ level or more. Ratios for main-sequence photospheres are indicated at the top right for stars with spectral types from M0 to B0.

monochromatic and in-band flux ratios of 4.325 and 4.558 , respectively. Thus our requirement for longer wavelength excesses to be relative to photospheric blackbody monochromatic flux ratios is the most conservative of the above set that could have been adopted.

It is important to be clear about what will be accepted or rejected by this simple algorithm, and to check that we are neither falsely accepting spurious IR-excess sources nor incorrectly rejecting true excess sources. Since we are simply taking ratios of flux densities and then comparing them to the ratios expected for a star of given spectral type, we can easily enumerate all conceivable outcomes. Note that in the following we refer to the continuum spectral energy distribution (SED) of a given star. In no case have we computed and fitted a SED to a set of FSC flux densities: we compare ratios of flux densities and are therefore not concerned with absolute values. We simply list each of the ways in which the FSC fluxes can, in principle, exhibit ratios that are either consistent or inconsistent with expectation for a stellar SED.

\subsubsection{Rejected Sources}

There are only three cases that can, in principle, lead to the observed and expected ratios being consistent, and both of the two cases that could actually occur in practice would justify rejection of a source (i.e., there should be no false rejections):

Case 1.-The FSC flux densities are consistent with expectation for the stellar SED. The ratios would, of course, also be consistent with expectation, so that the star would be correctly rejected by our test.

Case 2.-The observed flux density ratio is consistent with expectation, and all FSC flux densities are in fact significantly above the stellar SED at mid- and far-IR wavelengths. This can occur only if FSC fluxes are dominated by emission from a second source at a similar temperature, thereby excluding thermal emission from grains, which will be much cooler. The second source would most likely be a star, which could be either a companion to the star in question, or a field star within the IRAS beams. Again, our ratio test would correctly reject the source.

Case 3.- The observed flux density ratio is consistent with expectation, and the FSC flux densities are significantly below the stellar SED; although conceivable, this case cannot arise in practice since it would require a deficit in the stellar SED at mid-IR wavelengths.

\subsubsection{Accepted Sources}

Similarly, there are only three cases that can, in principle, lead to a significant difference between the observed and expected ratios, and both of the two cases that could actually occur in practice would demand that a source be accepted (i.e., there should be no falsely accepted sources):

Case 4.-A significant discrepancy exists between the observed and the expected flux density ratios, and the 12 , 25 , and $60 \mu \mathrm{m}$ FSC fluxes are in fact above the stellar SED. Assuming that the MSC and FSC sources are physically associated (and are not distinct sources that are coincidentally within the IRAS beam), the source is correctly accepted by our ratio test. The implication would also be that excess emission begins at a wavelength shortward of 12 $\mu \mathrm{m}$.

Case 5.-A significant discrepancy exists between the observed and expected flux density ratios, but the $12 \mu \mathrm{m}$ flux is consistent with the stellar SED. This implies that excess emission begins longward of $\lambda=12 \mu \mathrm{m}$, but the source would nonetheless be correctly accepted by our test. Similarly, for the (rare) case where FQUAL $=1$ at $12 \mu \mathrm{m}$ but there are good measurements at 25 and $60 \mu \mathrm{m}$, there could be a significant discrepancy between the observed and expected 25:60 $\mu \mathrm{m}$ ratios, while the $25 \mu \mathrm{m}$ flux is consistent with the stellar SED. This would imply excess emission beginning longward of $25 \mu \mathrm{m}$, but we would again accept the source correctly.

Case 6.-A significant discrepancy exists between the observed and expected flux density ratios, and one or more FSC flux densities are below the stellar SED. As for case 3 above, this scenario cannot arise in practice since it would again require a deficit in the mid-IR region of the photospheric SED.

\section{RESULTS}

Our selection criteria compel us to reject $99.9 \%$ of 112,971 stars in the MSC, leaving a total of 127 stars. $^{3}$ (The breakdown by MSC volume is 35 stars from Volume 1, 27 from Volume 2, 32 from Volume 3, and 33 from Volume 4.) Some 108 of these sources are candidate main-sequence stars with debris disks. (The remainder are classical $\mathrm{Be}$ stars: see below.) Of these, a subset of 73 stars were not identified during the previous searches summarized in $\S 2$. These "new" Vega-like sources are listed in Table 2. A total of 60 of these new stars exhibit discrepancies between observed and expected flux density ratios at greater than the $3 \sigma$ level. The 35 previously known MSC Vega-like stars are shown in Table 3. In Table 4 we list a further 12 stars

\footnotetext{
${ }^{3}$ In two instances, a single FSC source was found to be associated with two MSC stars that otherwise satisfied all the selection criteria; this occurred for the pair HD 24071 and HD 24072, and for the pair HD 35722 and HD 35736. These stars have been omitted.
} 


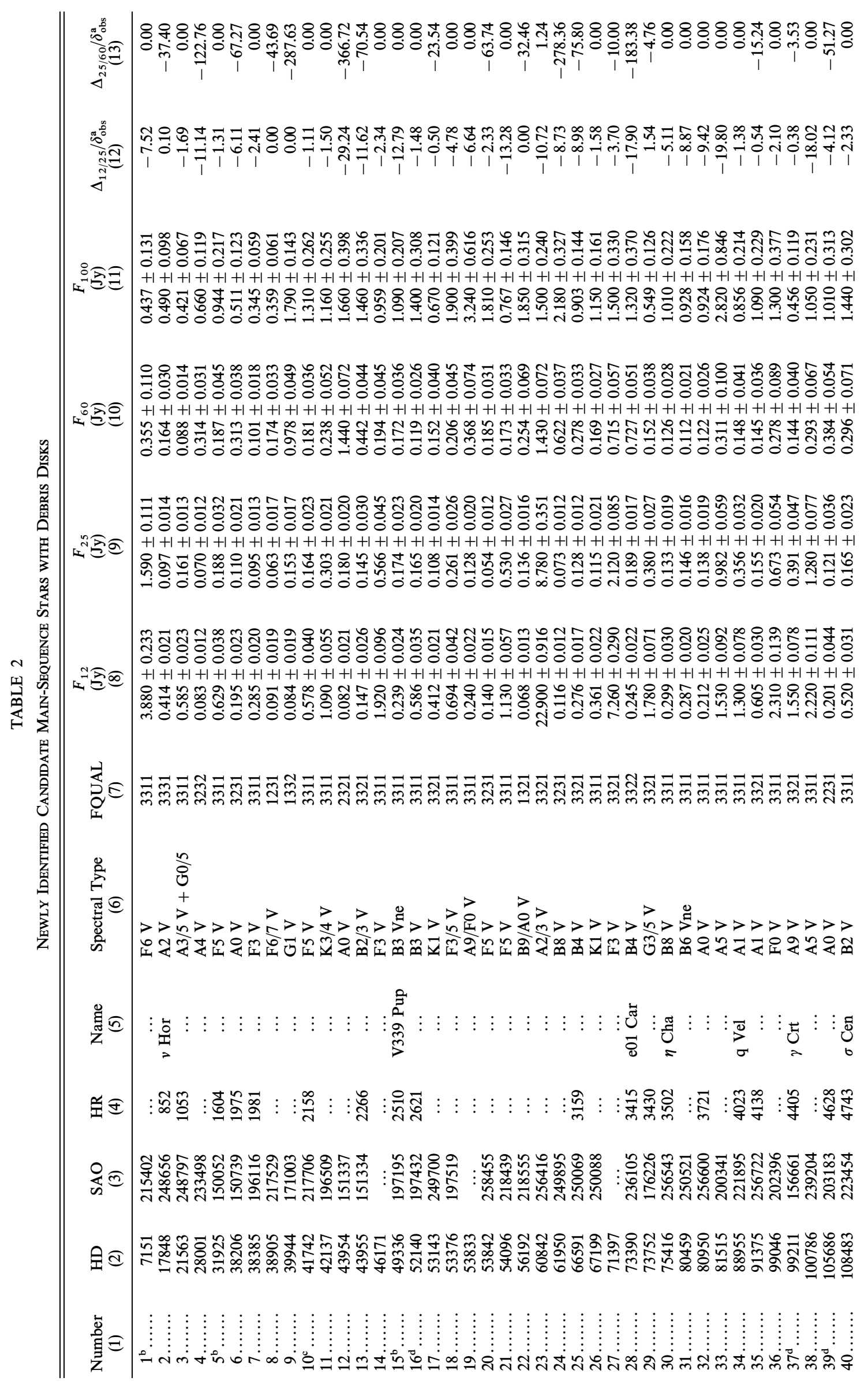




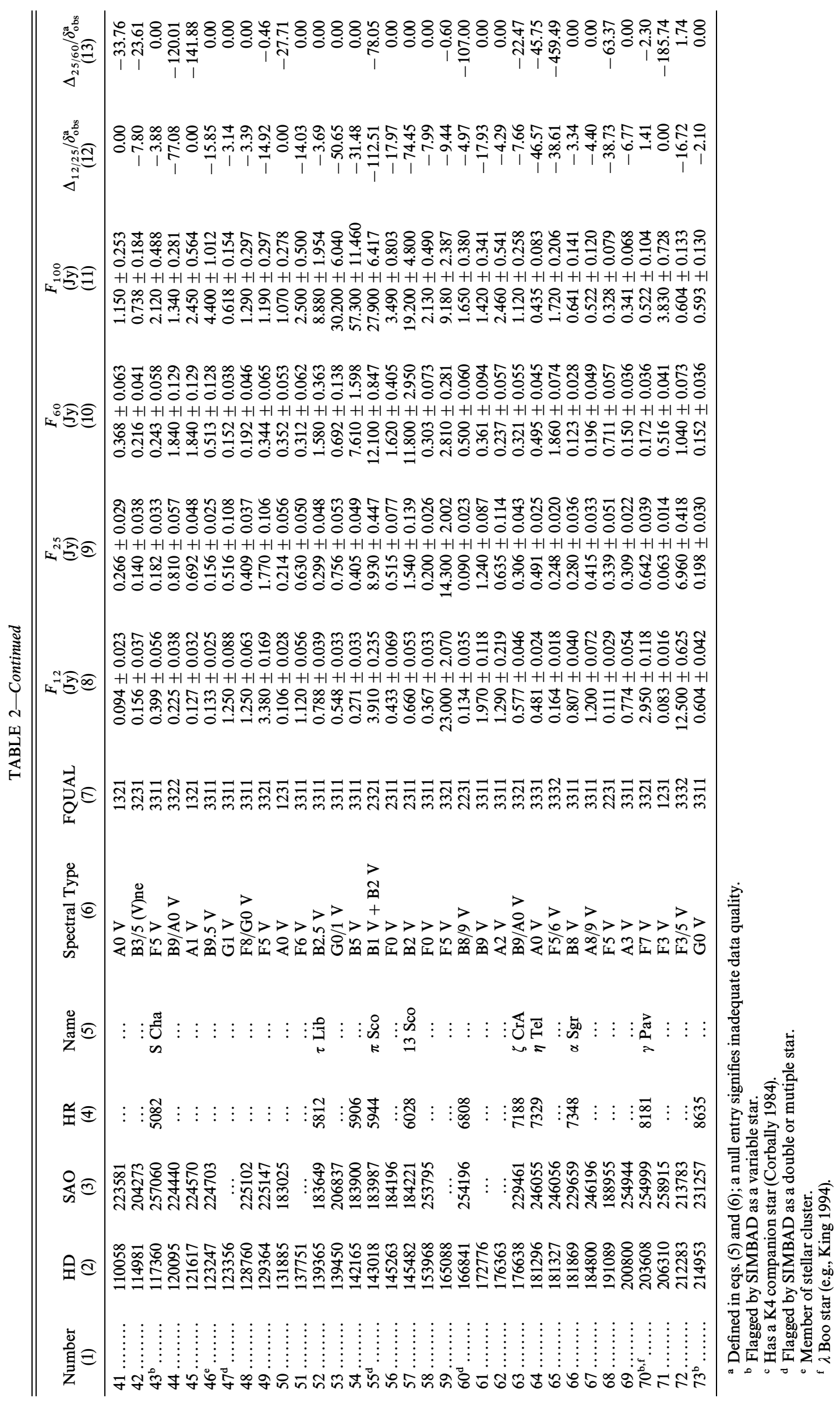




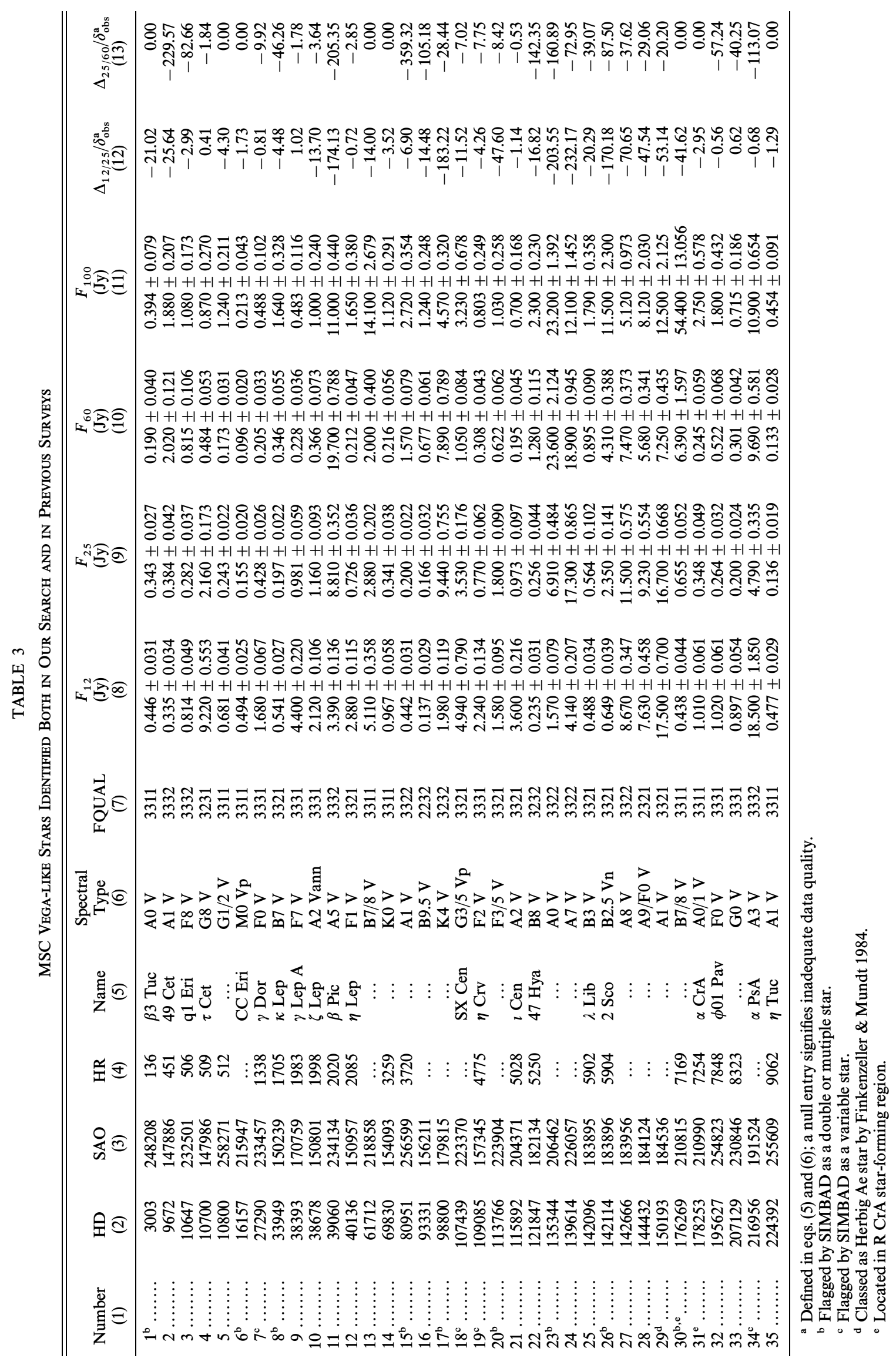




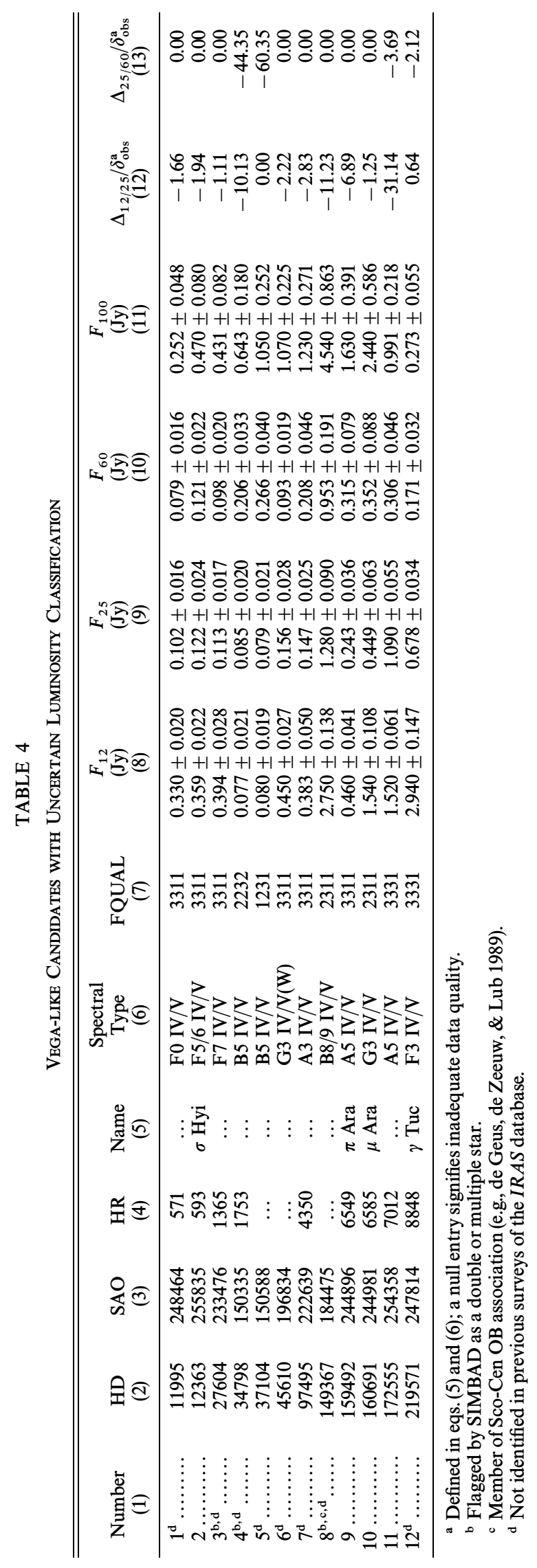




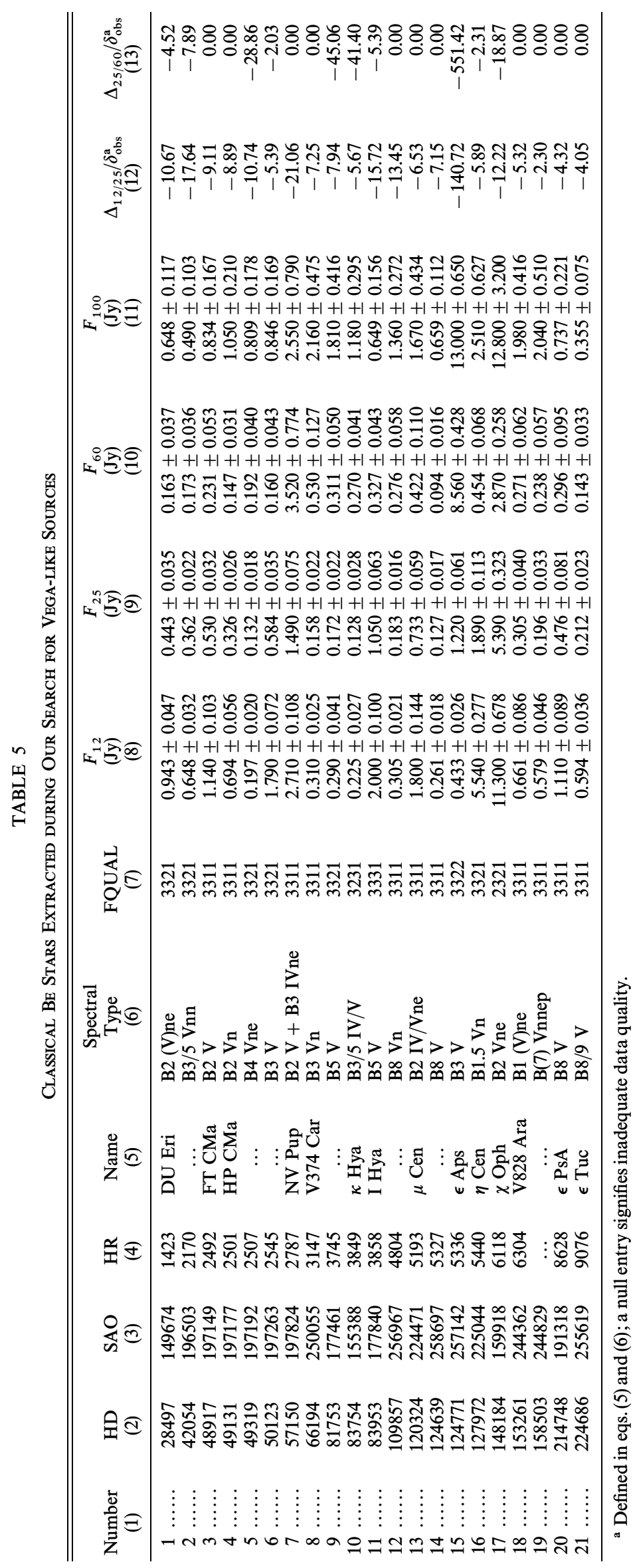


that satisfy all of our selection criteria, but where the assignment by the MSC of a luminosity class of V is uncertain. Stars labeled by footnote " $d$ " on their sequence numbers (col. [1] of Table 4) have not been identified in previous surveys of the IRAS catalogs. All other stars were found by one or more of the surveys listed in Table 1 . In Table 5 we show 19 classical Be stars of class $\mathrm{V}$ that were extracted during our search (and identified using the SIMBAD database), plus two Be sources with uncertain luminosity classifications. We will not consider the classical Be stars any further. Stellar proper motions are not included in the MSC, but we have used proper motions listed in the SAO catalog to examine the optical coordinates of the stars in Tables 2 and 4 a posteriori, and thereby check for spurious positional associations of MSC stars and FSC IR sources. Resultant proper motions range from $00^{\prime \prime} 1$ to $41^{\prime \prime}$, with a mean value of just 5 ".2 during the interval of time separating the epoch of the coordinates in the HD catalog (or, for MSC stars at declinations south of $-53^{\circ}$, the SAO catalog) and the epoch of the IRAS measurements. The separations of the proper-motion-corrected coordinates and the nominal coordinates of the FSC sources are less than $60^{\prime \prime}$ for all but two of the objects in Tables 2 and 4. The stars HD 184800 and HD 212283 are separated from their candidate FSC associations by 66.9 and $60 " .5$, respectively. Formally, they would be rejected by our selection criteria (which stipulate separations up to $\left.60^{\prime \prime}\right)$ but, since they exceed the search radius only marginally, we will retain them in our sample here. Some eight stars listed in Table 2 have no entry in the SAO catalog; in the absence of knowledge of their proper motions, these source candidates should be regarded with caution. Note that relatively nearby, high proper-motion sources could conceivably have been rejected mistakenly, since cumulative proper motions were not determined for the full set of MSC stars prior to positional association with the FSC. Our survey is therefore likely biased to the detection of the more distant debris disk sources.

In each of Tables 2 to 5 we provide, in ascending order of HD number, our sequence number (col. [1]), the SAO and HR numbers (where applicable), the name of the star, the MSC spectral classification, the quality flags for the four IRAS bands, and the FSC flux densities with $1 \sigma$ uncertainties. (Stellar coordinates are readily obtainable from on-line archives such as SIMBAD.) Columns (12) and (13) contain, for each star, our measures of the significance of the discrepancies between the observed mid- to far-infrared flux density ratios and the expected photospheric values, given the MSC spectral type. These columns correspond, respectively, to the values computed for the left-hand sides of the inequalities in equations (5) and (6) derived earlier: null entries are made when the quality flag of either the $12 \mu \mathrm{m}$ or the $25 \mu \mathrm{m}$ flux density is equal to unity. Included in the two Tables are only those stars for which inequalities in equations (5) and/or (6) hold. Such restriction to the $\geq 1 \sigma$ criterion is hardly conservative, but we include all such sources so that, should the reader prefer, a higher cutoff level can be adopted and a subset of the sources taken. We will discuss only the $\geq 3 \sigma$ sources below.

Note that we accept stars of all spectral types that meet the criteria described in $\S 3$, including 26 stars earlier than A0 with excesses $\geq 3 \sigma$. In the absence of any knowledge of the interstellar environment of these luminous stars we have made no attempt to exclude them, although the reader should keep in mind the possibility that at least some of the excess IR emission from these sources could be due to the heating of local interstellar material.

We made a further inspection of the 60 sources in Table 2 with discrepancies between observed and expected ratios above the $3 \sigma$ level. Each source was examined using the on-line IRSKY facility provided by the Infrared Processing and Analysis Center, which we employed to confirm all of the FSC flux densities obtained via our catalog manipulation, and to determine whether or not any of the stars also have positional associations with PSC objects. We found that $38 \%$ of the 60 stars ( 23 sources) have no entry in the PSC. Of the remainder, 13 stars have just one PSC measurement with an FQUAL flag $\geq 2$ at 12,25 , or $60 \mu \mathrm{m} ; 24$ stars have two or three measurements in these bands with FQUAL $>1$. There are many possible reasons why these latter stars were not identified during the surveys of the PSC. For example, 11 of the 24 stars have no entries in the SAO catalog and/or would have been filtered out by the criteria on FQUAL used by the two largest and most recent surveys, which we briefly discuss next.

\section{COMPARISON TO PREVIOUS METHODS}

We consider the methods used for two large surveys of the IRAS PSC by Stencel \& Backman (1991) and Oudmaijer et al. (1992).

To obtain their sample from the SAO catalog, Stencel \& Backman (1991) assumed that $12 \mu \mathrm{m}$ flux densities listed in the PSC were photospheric in origin, and a test was then made for IR excesses by comparing observed flux densities at 25,60 , and $100 \mu \mathrm{m}$ to values predicted using 12:25, 12:60, and 12:100 ratios for "normal" (dust-free) stars. As here, they used the errors on the flux densities to identify sources with significant excesses. However, to define photospheric ratios, they used median values of observed 12:25 $\mu \mathrm{m}$ ratios from their entire sample of position-associated stars, averaged over whole spectral type bands. These ratios were then used to derive equivalent color temperatures, with which 12:60 and 12:100 $\mu \mathrm{m}$ ratios were computed. Their 12:25 $\mu \mathrm{m}$ ratios for each spectral band (B, A, F, G, K, and M) turn out to be slightly less than would be predicted for blackbody emission. This is presumably due in part to the fact that some $7 \%$ of their sample of stars subsequently appear actually to have significant excess IR emission. They also speculate (cf., Cohen et al. 1987) that normal stars in general might have slight IR excesses.

Rather than attempt to determine photospheric ratios a priori and in a statistical fashion using a sample that would most likely include stars with circumstellar grains, we preferred to employ blackbody ratios, source-by-source, for each spectral subclass. We computed the photospheric ratios using the relatively accurate spectral types assigned by the MSC; Stencel \& Backman (1991) adopted the less reliable spectral types listed in the SAO catalog, hence their use of whole spectral bands, although it must be noted that at far-IR wavelengths there is in fact little spread in the ratios across any given band. Note also that, since Stencel $\&$ Backman used the $12 \mu \mathrm{m}$ flux density only to scale the predicted flux densities at 25,60, and $100 \mu \mathrm{m}$ and probe for excess emission in these latter bands, their sample could include stars with or without excess emission at $12 \mu \mathrm{m}$.

However, the Stencel \& Backman (1991) technique could lead to some stars with genuine IR excesses nevertheless being rejected. First, a $12 \mu \mathrm{m}$ flux that is in fact in excess of the photospheric value for a given star would of course lead 
to an overestimation of the predicted photospheric $25 \mu \mathrm{m}$ flux: it could sometimes happen that the flux measured at $25 \mu \mathrm{m}$ is in reality also significantly above the photospheric level, but nonetheless at or below the high predicted value, which would lead to a false rejection of the star. We avoid this possibility in the present work by taking flux ratios, rather than using absolute values. Second, the demand for a $12 \mu \mathrm{m}$ detection obviously excludes all sources that are detected with moderate or excellent quality only at 25 and $60 \mu \mathrm{m}$; such sources account for $6 \%$ of our final sample in Tables 2 and 3. A comparable fraction may have been missed by Stencel \& Backman (1991).

Oudmaijer et al. (1992), like Stencel \& Backman (1991), cross-correlated the SAO catalog with the IRAS PSC and searched for IR excesses from stars of various spectral types and luminosity classes. However, instead of comparing IRAS flux densities or flux density ratios directly, Oudmaijer et al. (1992) derived 12,25 , and $60 \mu \mathrm{m}$ magnitudes, after which they inspected the resulting scatter in a $[25]-[60],[12]-[25]$ diagram and searched for avoidance of the locus defined by photospheric colors.

\section{DISCUSSION}

In Figure 1 we plot $F_{25} / F_{60}$ against $F_{12} / F_{25}$ for the subset of 50 of the 108 new and previously identified Vegalike stars (Tables 2 and 3 ) that have excellent- or moderatequality detections in all three of the 12, 25, and $60 \mu \mathrm{m}$ bands, and for which at least one of the flux density ratios deviates from the computed photospheric value by $3 \sigma$ or more. The (short) locus for blackbody main-sequence photospheres is drawn at the top right of this diagram. Note that the handful of data points (four stars) with "forbidden" excursions either above or to the right of the main-sequence locus are nevertheless within 1 to $2 \sigma$ of it. Also, not surprisingly, given the proximity of all photospheric mid-IR SEDs to Rayleigh-Jeans curves, it becomes clear that the assumption in equation (7) concerning errors on the stellar spectral types is irrelevant for these sources, since they all have at least one ratio that avoids the entire main-sequence locus.

Two of the prototype Vega-like stars, $\beta$ Pic and Fomalhaut ( $\alpha$ PsA), were extracted during our search, and it can be seen that they both lie in well-populated regions of Figure 1: evidently, their mid- and far-IR colors are not unusual.

In Figure 2 we show a histogram of the 88 new and previously recognized MSC Vega-like stars with at least one flux density ratio that is discrepant with the photospheric value at the $3 \sigma$ level or higher. The abscissa is stellar effective temperature $\left(T_{\text {eff }}\right)$, and the sources are grouped into bins of width $0.1 \mathrm{dex}$. MSC spectral types were converted to temperatures using the calibrations by Gray \& Corbally (1994). (Spectral types are shown along the top of Fig. 2.) The distribution of sources is seen to rise quickly with decreasing $T_{\text {eff }}$, until we reach early A-type stars. There is a dip for late A stars (reflecting the smaller number of stars classified as such) followed by a second peak around G0, below which the distribution cuts off $\left(T_{\text {eff }}<4500 \mathrm{~K}\right)$. The latter characteristic is most likely due to the limiting sensitivity of the measurements in the FSC rather than to a paucity of debris disks around stars of spectral type mid-G and later. Combining the data of Tables 2, 3, and 5, we find that 43 stars with spectral types earlier than B8 are selected by our criteria, of which 21 are classical Be stars (Table 5) with free-free excesses. The remaining B stars (in Tables 2

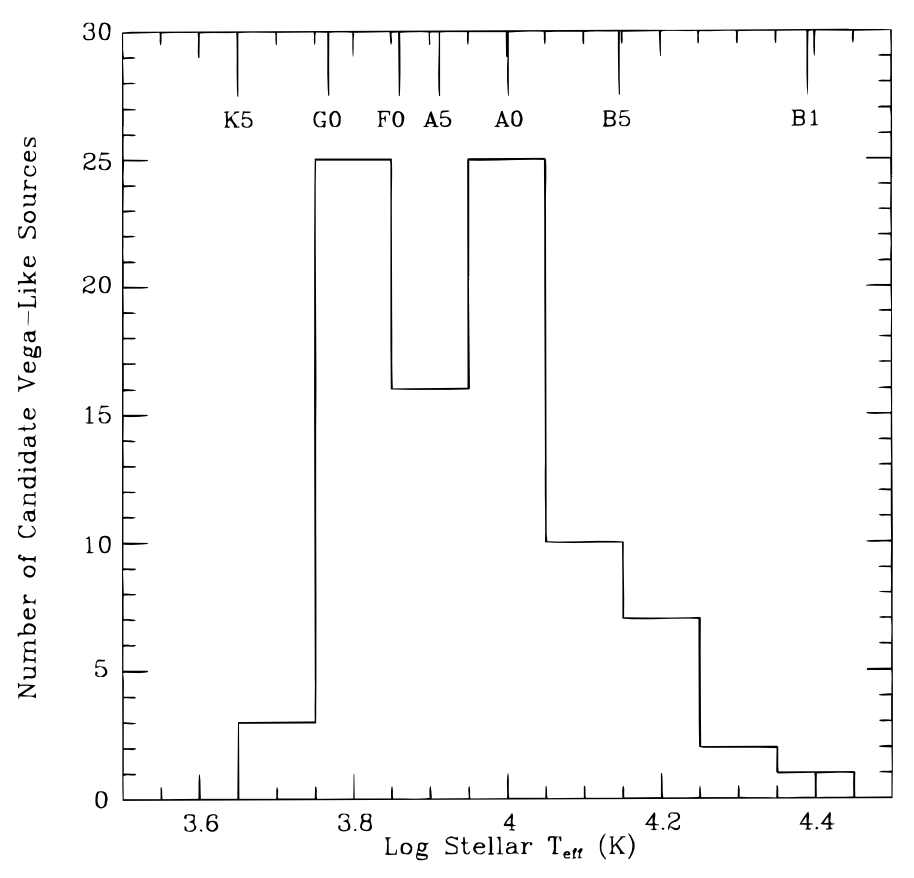

Fig. 2.-Histogram of the number of stars versus stellar effective temperature, for new and previously identified sources with at least one $I R A S$ flux ratio that is discrepant by $3 \sigma$ or more with the expectation for a blackbody of the corresponding stellar effective temperature.

and 3) show excesses attributable to dust emission, although in the case of some of the more luminous early B-type stars (e.g., $\pi$ Sco, 2 Sco, 13 Sco), this emission may arise from warm dust in surrounding reflection nebulae.

\section{CONCLUSIONS}

We have used the positions and luminosity classes of Southern Hemisphere sources listed in the Michigan Catalog of Two-dimensional Spectral Types for the HD Stars together with the positions, data-quality flags, and flux densities of mid- and far-IR sources in the IRAS Faint Source Survey Catalog to isolate a sample of main-sequence stars with possible excess IR emission, most likely indicative of thermal radiation from dust grains in associated debris disks. Our search criteria extracted a total of 108 mainsequence stars with possible IR excesses, 73 of which have not been previously identified. For some 60 of these "new" candidate Vega-like stars, measured IR flux density ratios and expected photospheric ratios are discrepant above the 3 $\sigma$ level. Further analysis of a subset of the new sources will be made following near- to far-IR photometry with the Infrared Space Observatory and ground-based optical and IR photometry and spectroscopy.

V. M. was supported during the initial stages of this work by the UK Particle Physics and Astronomy Research Council, and currently by NASA grant NAGW-4030 from the Origins of Solar Systems program. We have made extensive use of the on-line facilities of the National Space Science Data Center at the NASA Goddard Space Flight Center, together with the SIMBAD database operated at CDS, Strasbourg, France. Many thanks are due to Anneila Sargent and Dana Backman for reading our manuscript. We also thank Alan Wood of the Rutherford Appleton Laboratory, UK, for his quick and helpful responses to our queries concerning the manipulation of data catalogs. We thank R. Oudmaijer and an anonymous referee for their comments on the paper. 


\section{REFERENCES}

Aitken, D. K., Moore, T. J. T., Roche, P. F., Smith, C. H., \& Wright, C. M. 1993, MNRAS, 265, L41

Aumann, H. H. 1985, PASP, 97, 885

Aumann, H. H., et al. 1984, ApJ, 278, L23

Aumann, H. H., \& Probst, R. G. 1991, ApJ, 368, 264

Backman, D. E., \& Paresce, F. 1993, in Protostars and Planets III, ed. E. H. Levy \& J. I. Lunine (Tucson: Univ. Arizona Press), 1253

Cheng, K. P., Bruhweiler, F. C., Kondo, Y., \& Grady, C. A. 1992, ApJ, 396, L83

Chini, R., Krügel, E., Shustov, B., Tutukov, A., \& Kreysa, E. 1991, A\&A, 252,220

Cohen, M., Schwarz, D., Choksi, A., \& Walker, R. 1987, AJ, 93, 1199

Cohen, M., Walker, R. G., Barlow, M. J., \& Deacon, J. R. 1992, AJ, 104, 1650

Corbally, C. J. 1984, ApJS, 55, 657

Coté, J. 1987, A\&A, 181, 77

de Geus, E. J., de Zeeuw, P. T., \& Lub, J. 1989, A\&A, 216, 44

Finkenzeller, U., \& Mundt, R. 1984, A\&AS, 55, 109

Gillett, F. C. 1986, in Light on Dark Matter, ed. F. P. Israel (Dordrecht: Reidel), 61

Gliese, W. 1969, Catalog of Nearby Stars (Heidelberg: Publ. Astr. Rechen Institute)

Gray, R. O., \& Corbally, C. J. 1994, AJ, 107, 742

Houk, N. 1978, Michigan Catalog of Two-dimensional Spectral Types for the HD Stars, Vol. 2 (Ann Arbor: Univ. Michigan)

. 1982, Michigan Catalog of Two-dimensional Spectral Types for the HD Stars, Vol. 3 (Ann Arbor: University of Michigan)

. 1994, in PASP Conf. Ser. 60, The MK Process at 50 Years: A Powerful Tool for Astrophysical Insight, ed. C. J. Corbally, R. O. Gray, \& R. F. Garrison (San Francisco: ASP), 285

Houk, N., \& Cowley, A. P. 1975, Michigan Catalog of Two-dimensional Spectral Types for the HD Stars, Vol. 1 (Ann Arbor: Univ. Michigan)

Houk, N., \& Smith-Moore, M. 1988, Michigan Catalog of Twodimensional Spectral Types for the HD Stars, Vol. 4 (Ann Arbor: Univ. Michigan)

IRAS Catalogs and Atlases: Explanatory Supplement. 1988, ed. C. A. Beichman, G. Neugebauer, H. J. Habing, P. E. Clegg, \& T. J. Chester (Washington, DC: GPO)
IRAS Point Source Catalog, Version 2. 1988, Joint IRAS Science Working Group (Washington, DC: GPO) (PSC)

Jaschek, M., Jaschek, C., \& Egret, D. 1986, A\&A, 158, 325

Johnson, H. 1986, ApJ, 300, 401

Kalas, P., \& Jewitt, D. 1996, AJ, 111, 1347 1997, Nature, 386, 52

King, J. R. 1994, MNRAS, 269, 209

Knacke, R. F., Fajardo-Acosta, S. B., Telesco, C. M., Hackwell, J. A., Lynch, D. K., \& Russell, R. W. 1993, ApJ, 418, 440

Lagrange-Henri, A. M. 1995, Ap\&SS, 223, 19

Moshir, M., et al. 1989, Explanatory Supplement to the IRAS Faint Source Survey (Pasadena: JPL) (FSC)

Odenwald, S. F. 1986, ApJ, 307, 711

Oudmaijer, R. D., van der Veen, W. E. C. J., Waters, L. B. F. M., Trams, N. R., Waelkens, C., \& Engelsman, E. 1992, A\&AS, 96, 625

Patten, B. M., \& Willson, L. A. 1991, AJ, 102, 323

Sadakane, K., \& Nishida, M. 1986, PASP, 98, 685

Skinner, C. J., Barlow, M. J., \& Justtanont, K. 1992, MNRAS, 255, 31P

Skinner, C. J., Sylvester, R. J., Graham, J. R., Barlow, M. J., Meixner, M., Keto, E., Arens, J. F., \& Jernigan, J. G. 1995, ApJ, 444, 861

Smith, B. A., Fountain, J. W., \& Terrile, R. J. 1992, A\&A, 261, 499

Smith, B. A., \& Terrile, R. J. 1984, Science, 226, 1421

Stencel, R. E., \& Backman, D. A. 1991, ApJS, 75, 905

Sylvester, R. J., Barlow, M. J., \& Skinner, C. J. 1994a, MNRAS, 266, 640

. 1994b, Ap\&SS, 212, 261

Sylvester, R. J., Skinner, C. J., Barlow, M. J., \& Mannings, V. 1996, MNRAS, 279, 915

Telesco, C. M., \& Knacke, R. S. 1991, ApJ, 372, L29

Vidal-Madjar, A., \& Ferlet, R. 1994, in Circumstellar Dust Disks and Planet Formation, ed. R. Ferlet \& A. Vidal-Madjar (Paris: Editions Frontières), 7

Walker, H. J., \& Wolstencroft, R. D. 1988, PASP, 100, 1509

Zuckerman, B., \& Becklin, E. E. 1993, ApJ, 414, 793 